\section{Increased risk of stroke mortality in nondiabetic individuals with hyperglycemia}

Nondiabetic individuals with elevated bloodglucose levels could be at increased risk of stroke. The Diabetes Epidemiology: Collaborative Analysis of Diagnostic Criteria in Europe (DECODE) study group has, therefore, assessed the potential relationship between hyperglycemia and stroke mortality, and compared two diagnostic criteria for stroke-risk prediction.

Hyvärinen and colleagues enrolled 21,706 individuals (55\% of whom were men) from 13 European cohorts, in which the mean age ranged from 45 to 76 years. Median duration of follow-up was 10.5 years. Diabetes mellitus had previously been diagnosed in $5.5 \%$ of the study population. Hyperglycemia was defined by the fasting plasma-glucose (PG) level or the $2 \mathrm{~h}$ post-challenge $P G$ level in participants without previously diagnosed diabetes mellitus.

The risk of stroke mortality was increased in participants with known diabetes mellitus. The risk was also increased for individuals with hyperglycemia, regardless of the diagnostic criteria used. A multivariate analysis demonstrated that a 1 SD increase in fasting PG corresponded to a hazard ratio of 1.02 in men and 1.52 in women. Similarly, a $1 \mathrm{SD}$ increase in $2 \mathrm{~h}$ PG corresponded to a hazard ratio of 1.21 in men and 1.31 in women. Waist circumference and triglyceride levels did not significantly affect risk prediction.

The authors conclude that for individuals without a previous diagnosis of diabetes mellitus, an elevated $2 \mathrm{~h} \mathrm{PG}$ level is the best predictor of stroke mortality in men, whereas an elevated fasting PG level is the best predictor in women.

Original article Hyvärinen M et al. (2008) Hyperglycemia and stroke mortality - comparison between fasting and

2-hour glucose criteria. Diabetes Care [doi:10.2337/dc08-1411]

\section{Imatinib shows promise for the treatment of type 1 diabetes mellitus}

The development of small-molecule tyrosinekinase inhibitors, such as imatinib, has transformed cancer chemotherapy. Louvet and colleagues questioned whether these agents might also be effective in the treatment of autoimmune disorders, such as type 1 diabetes mellitus.

The authors treated nonobese, diabetic (NOD) mice with $1.5 \mathrm{mg}$ imatinib or peanut oil once daily. Treatment with imatinib completely inhibited the development of diabetes mellitus in prediabetic NOD mice. By contrast, around $40 \%$ of prediabetic control mice developed diabetes mellitus. After cessation of treatment, diabetes mellitus developed in $20 \%$ of the imatinib-treated animals and in $71 \%$ of the control animals by 30 weeks of age. Treatment with imatinib was also found to reverse disease in NOD mice with new-onset diabetes mellitus. Furthermore, long-lasting remission occurred after a relatively short treatment period (10 weeks).

The precise mechanisms of the effect of imatinib on diabetes mellitus remain to be uncovered. Imatinib did not alter insulinitis, T-cell number or antigen-specific T-cell proliferation in NOD mice. One possibility that warrants further investigation, however, is specific inhibition of the platelet-derived growth-factor receptor.

Studies of imatinib in humans for the treatment of rheumatoid arthritis and Crohn disease are currently in progress. In light of their findings in the NOD mouse, Louvet et al. suggest that imatinib might also be considered as a potential therapy for patients with type 1 diabetes mellitus.

Original article Louvet C et al. (2008) Tyrosine kinase inhibitors reverse type 1 diabetes in nonobese diabetic mice. Proc Natl Acad Sci USA 105: 18895-18900

\section{Both genes and environment contribute significantly to BMI}

Studies in biological twins have estimated the genetic component of BMI, but the contribution of environmental factors remained uncertain. To differentiate between the genetic and environmental influences on BMI, Segal et al. conducted a study that included natural twins and virtual twins (i.e. same-age siblings, one or both of whom were adopted in infancy).

The 929 participants included 164 monozygotic twin pairs, 156 dizygotic twin pairs, 5 sets of triplets, 1 set of quadruplets, 128 virtual twin pairs, 2 sets of virtual triplets and 2 sets of virtual quadruplets. A linear, mixed model was 